$\xi=$ 不

\title{
Geophysical study in En Nuhud basin area
}

\author{
Asim Abubabakr Elmansour ${ }^{1 *}$, Omar Attag Omar ${ }^{2}$ \\ ${ }^{1}$ Department of Geology, Faculty of Science, University of Kordofan, Sudan \\ ${ }^{2}$ Department of Geolog, Faculty of Petroleum and Mining, University of El Neelain, Sudan \\ *Corresponding author E-mail: umdaballo63@gmail.com
}

\begin{abstract}
Integration of gravity, magnetic, resistivity and geophysical well logging methods were used to reveal the geological structures and the hydrogeological conditions. Magnetic mineralogy among the primary bed rocks or within the sedimentary sequence considerably affect the responses of gravity and magnetic methods. En Nuhud Basin is structural depression as a half-graben produced by displacement along a system of faults. Gravity measurements give a sense of that the area was dissected by different fault trends of NNW-SSE and E$\mathrm{W}$ trends, with down throws towards the center of the basin, and variable magnitudes of displacement. The thickness of the sediments occupying the basin vary from few meters at the periphery of the basin to about 1000 meters at the southern parts. The water saturated zones in the Nubian Formation show good characteristics and a thickness range from 12- $140 \mathrm{~m}$. The weathered Basement Complex is sometimes serve as aguiferous zone.
\end{abstract}

Keywords: En Nuhud Basin; Half-Graben; Nubian Formation; Gravity; Magnetic; Resistivity; Well Logging.

\section{Introduction}

The study area is situated in west-central Sudan, within Kordofan Region and covering an area of about $20,466 \mathrm{~km}$. It is bounded by longitudes $28^{\circ} 15^{\prime}$ and $30^{\circ} 00^{\prime}$, and latitudes $12^{\circ} 22^{\prime}$ and $13^{\circ} 28^{\prime}$ (Fig. 1).

Many studies were conducted in the dealing with different geological aspects such as Rodis et al (1964), Karkanis, (1966), Strojexport (1971-1976), Ginaya, (2001) and Ginaya, (2011). Complexes of geophysical methods were used in this study, those are: gravimetric method, surface geo-electric method, magnetometric method and geophysical well-logging. Geophysical, as well as borehole data, were compiled from different agencies and corporations, in addition to the field work for this study.

Geophysical measurements were performed at supposed tectonic or transgressive geologic contacts, or where the depth to the Basement Complex is not known. Correlation of geophysical measurements with borehole logs was performed and hence construction and tectonic classification of hydrogeological structures were achieved.

\section{Objectives}

Geophysical methods were used to examine the geological structures and the hydrogeological conditions in the study area.

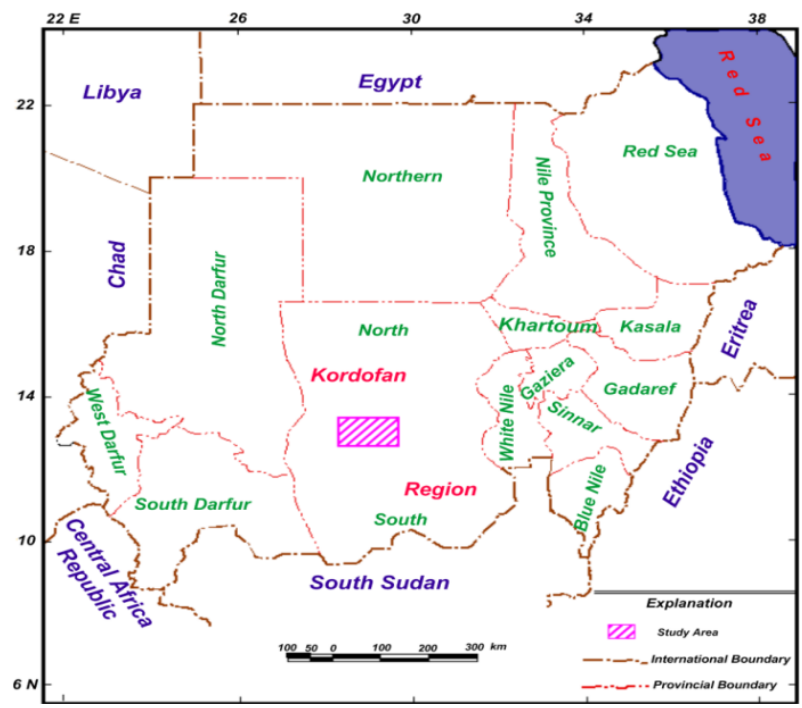

Fig. 1: Location Map of the Study Area.

\section{Materials and methods}

Gravity measurements were used for determination of the relief and depth of the Basement Complex, and so the thickness of the sedimentary series and/or their structural- tectonic relationship to the Basement. These goals were planned to be established under the consideration that the density contrast $\Delta \delta$ between the two environments is constant. When the mentioned condition is usually not fulfilled, only lithological boundary as a density (not stratigraphic) boundary is traced. Magnetometry was used to trace magnetic rock environments and to assist in eliminating the ambiguity in interpretation of gravity measurements.

Resistivity method is applied to determine the characteristics of the hydrogeological units in the study area. The vertical electrical 
sounding (VES) technique is used to investigate the change in resistivity with depth, where it is a function of rock type, mineralogical composition, moisture content, and other conditional factors. The limitation of this method is the assumption of a horizontally homogeneous medium and the uniform thickness of the layered rocks (Dobrin, 1988). Based on the fact that the depth of penetration of the method is limited by the maximum electrical power that can be introduced into the ground, and the depth resolution of resistivity method usually decreases with increasing depth (Keary et al, 2002). The method was used only to give information within a depth of 200-300 meters.

Geophysical logs in the study area were executed where loss of fluid circulation occurs, as resistivity (long normal), self potential and drilling time logs. As the interpretation of geophysical logs usually influenced by the numerous environmental factors causing log response that difficult to analyze quantitatively, in addition to the lack of adequate data to guide log interpretation (due to loss of circulation), and the limited types of geophysical logs, the logs were interpreted mainly qualitatively, to determine lithology and geometry of aquifer system, and to estimate the quality of contained water.

\section{Geology}

En Nuhud Basin is a rift structure filled with a thick sedimentary sequence. The main rock units in the area are the Basement Complex, Nubian Sandstone Formation, Laterites and the Superficial Deposits (Fig. 2).

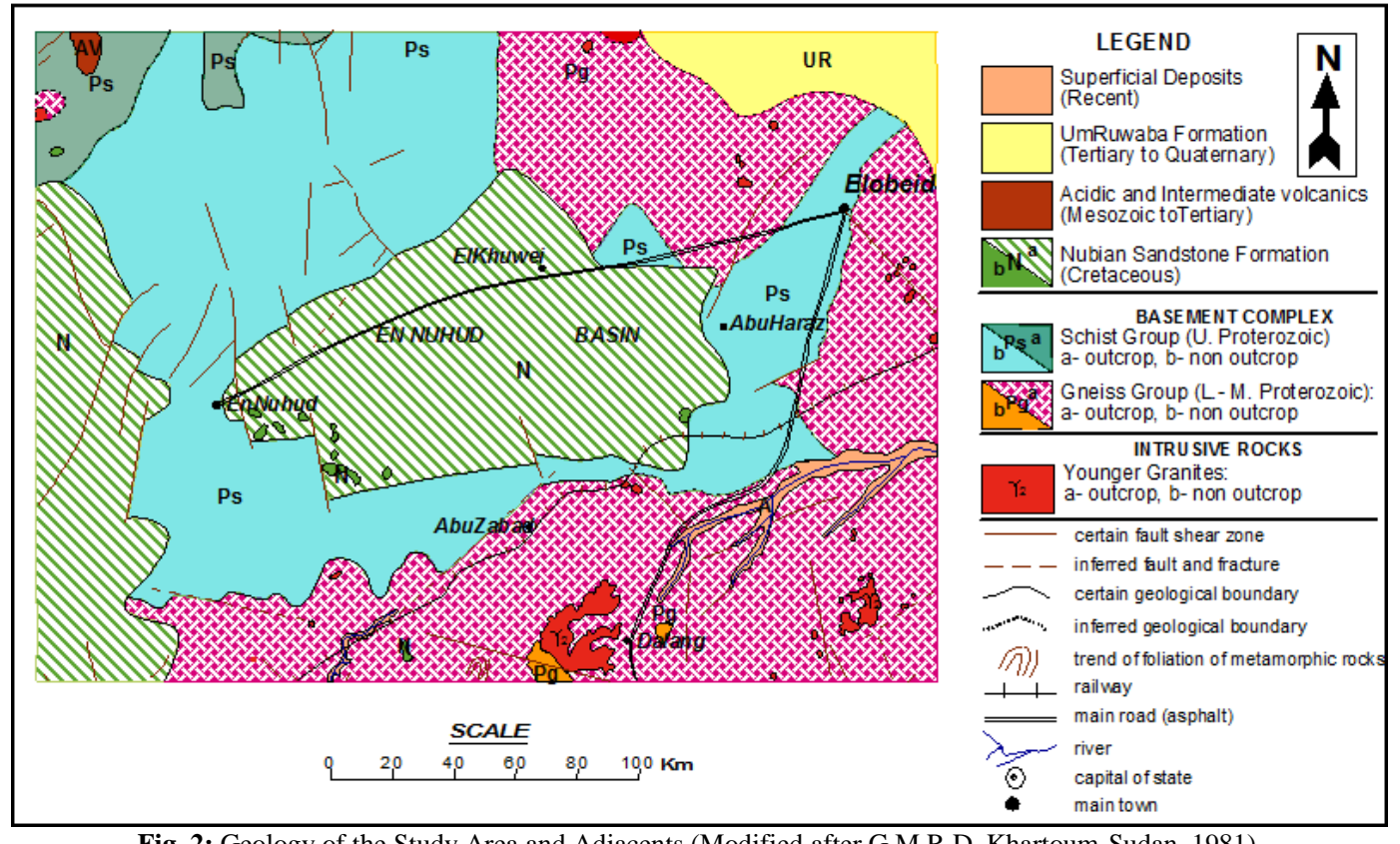

Fig. 2: Geology of the Study Area and Adjacents (Modified after G.M.R.D, Khartoum-Sudan, 1981).

Basement Complex is the oldest rocks, most of which are Precambrian in age. It is largely undifferentiated in much of the area and includes metamorphosed and folded schists and gneisses, as well as syn-orogenic or late orgoenic granitic emplacements and basic and ultrabasic bodies. Included in the Basement Complex are postorogenic younger intrusive, Possibly early Palaeozoic (Vail 1978).

The Nubian Sandstone Formation represents the Mesozoic Formations in Kordofan region (Rodis et al, 1964). The common lithology is poorly sorted, coarse to medium grained sandstones and conglomerates, containing quartz pebbles and mud flakes; cross- bedding, graded- bedding and rapid facies changes are common. The mudstones and clays are usually thinly bedded and do not form conspicuous outcrops (Vail, 1978). For the age of the Cretaceous Sandstone Formation in Sudan, Whiteman (1971) allocate Early cretaceous in the base of fossil evidence; Harms et al (1990) categorized it as Upper Jurassic/ Cretaceous.

The laterite consists of a highly ferruginous layer ironstones. Limited erosional remnants of these deposits occur in the western part of the study area. Geologists proposed ages extended from late cretaceous to early Tertiary (Whiteman, 1971); Rodis etal (1964) proposed an age of Early to Middle Tertiary time for these deposits in Kordofan province.

The Superficial Deposits are of Pleistocene and Recent ages and include Qoz sands, clay plains, hill wash deposits and alluvia deposits. Qoz Sands forms gently-rolling sheets and fixed dunes (Whiteman, 1971). Clay plains are remarkably uniform clay deposits, with $(50-60 \%)$ clay content and very little coarse material Rodis et al (1964). Wash deposits occur along foot slopes of jebels as unsorted coarse-grained clastic material, (Strojexport, 1976).
Alluvial deposits are placed along the water courses as unconsolidated sands, clays and gravels.

\section{Geophysical measurements and processing}

\subsection{Gravimetric}

Gravity measurements cover approximately the western half of the study area, with individual gravity stations spaced at $1000 \mathrm{~m}$ or $500 \mathrm{~m}$ interval. Altitude of stations was determined with an error equal $\pm 12.9 \mathrm{~cm}$ and the precision of individual gravity station measurements is estimated as $\pm 0.03 \mathrm{mgal}$. Densities on a limited number of samples from rock outcrops around the study area were measured in laboratory. Density values are proposed as $\delta=2.2$ $\mathrm{g} / \mathrm{cm}^{3}$ for the sedimentary series and $2.7 \mathrm{~g} / \mathrm{cm}^{3}$ for the Basement Complex, then $\Delta \delta=0.5 \mathrm{~g} / \mathrm{cm}^{3}$ is obtained. The measured gravity data is reduced to Bouguer, and then profiles and maps were constructed.

\subsection{Geomagnetic}

The "z" component of the geomagnetic field was measured with $500 \mathrm{~m}$ station step and reading precision sensitivity 12.5 gamma. Repeated measurements as reference points were carried out to improve the quality of the measurements. The mean square error had been calculated from the results of repeated measurements and the precision was \pm 6.8 


\subsection{Geoelectric}

A total of (79) VESs were performed in the study area (Fig. 3), (21) of which were concentrated in Hydoub well field, the more potential zone in the basin area. ABEM, SAS 1000 instrument is used, where schlumberger electrode array is applied with maximum spacing ranges from 200 to $900 \mathrm{~m}$. Some VESs were performed at boreholes for calibration of resistivity measurements. Resistivity data were processed using IX1D software. Correction factor for the investigated depth is calculated using the lithologic logs of some boreholes as follows:

Correction Factor $\left(\mathrm{CF}_{\mathrm{d}}\right)=\frac{\text { Actual Depth }}{\text { Calculated Depth }}$
Then a mean value of 0.655 for $\mathrm{CF}_{\mathrm{d}}$ is obtained.

The formation water resistivity $\mathrm{R}_{\mathrm{w}}$ is calculated using the "EC" values measured in the field for some boreholes. Based on Keys et al (1981), the $R_{w}$ is obtained from the relation, as follows:

$\mathrm{R}_{\mathrm{w}}=\frac{10000}{\mathrm{EC}}$

According to Archie's law (Kirsch, 2006), the resistivity of water saturated clay-free material can be described as:

$\mathrm{R}_{\mathrm{f}}=\mathrm{R}_{\mathrm{w}} \cdot \mathrm{F}$

Where " $\mathrm{R}_{\mathrm{f}}$ is the formation resistivity and " $\mathrm{F}$ " is the formation Factor.

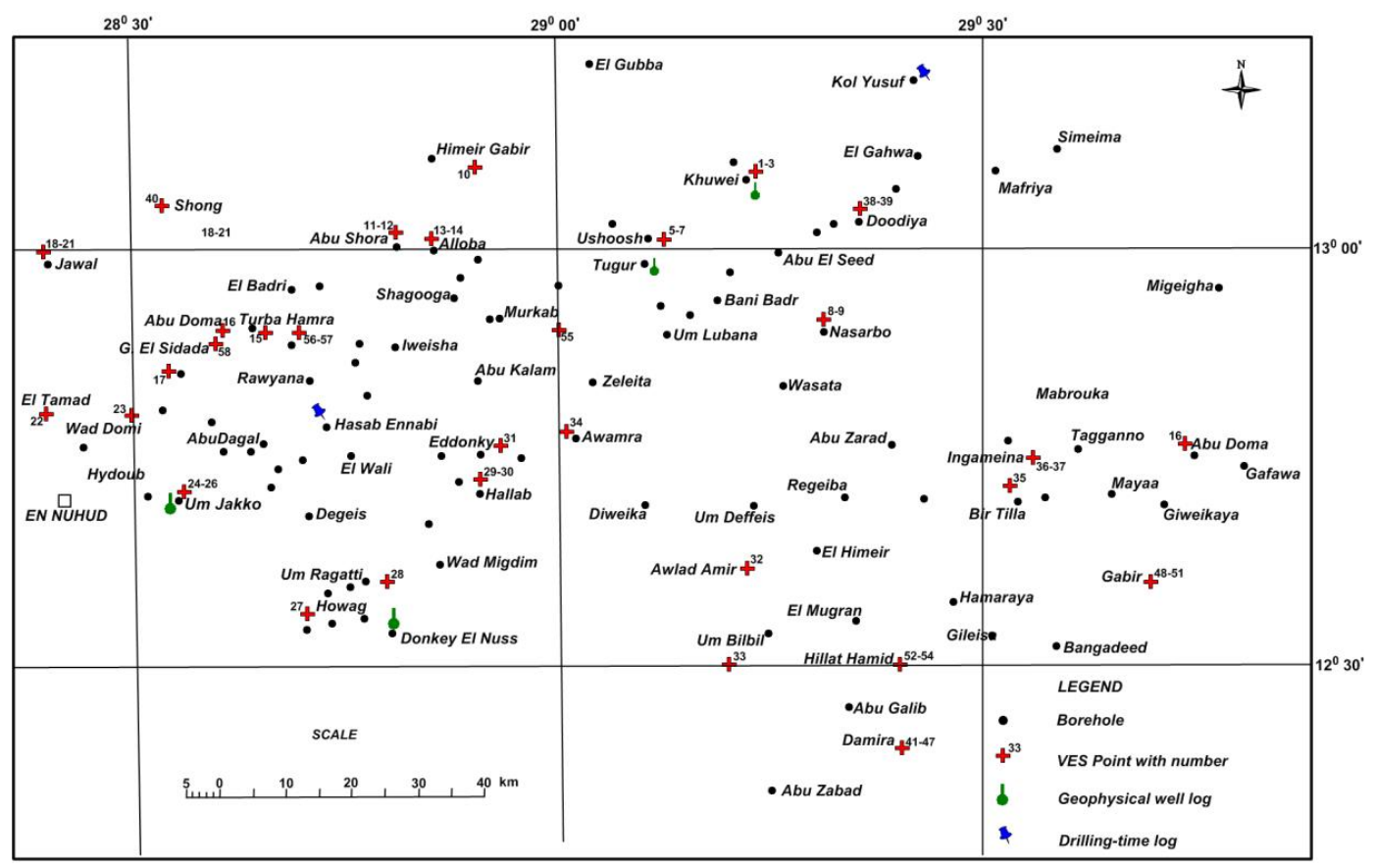

Fig. 3: Locations of Vess and Geophysical Well Logs.

Using the apparent resistivity standing for the formation resistivity (generally ranges from 18 to $480 \mathrm{ohm} . \mathrm{m}$ ), then, the (F) is calculated as:

$\mathrm{F}=\frac{\mathrm{R}_{\mathrm{f}}}{\mathrm{R}_{\mathrm{w}}}$,

Also according to Archie's law:

$\mathrm{F}=\mathrm{a} \phi^{-\mathrm{m}}$

Where "a" is an empirical constant related to zone of interest $(0.62$ $<\mathrm{a}<1.0$ ), and " $\mathrm{m}$ " is cementation factor, which depends on grain size and tortuosity of pore spaces $(2.0<\mathrm{m}<3.0)$, and " $\phi "$ is the porosity of the saturated zone (Keary et al, 2002).

For the typical sandstones of oil reservoirs Archie found that the coefficient "a", was close to one and the exponent " $\mathrm{m}$ " was close to two. Subsequent work with other rocks and even unconsolidated sediments showed that this power law is generally valid, but with varying coefficients and exponents. Keller (1996) summarizes the coefficients for different materials (table 1).

\subsection{Geophysical well logs}

Few geophysical logs were executed at boreholes in the study area (Fig. 3), at locations where loss of fluid circulation occurs. Geophysical logs in four boreholes as resistivity (long normal) and self potential logs, and two as drilling time logs, were examined for the physical characteristics of the subsurface environment.
Table 1: Summary of the Coefficients in Archie's Formula for Different Materials (According To Keller (1996))

\begin{tabular}{llll}
\hline Rock & $\phi$ & $\mathrm{a}$ & $\mathrm{m}$ \\
\hline Weakly cemented, detrital (Tertiary) & $0.25-0.45$ & 0.88 & 1.37 \\
Moderately well cemented (Mesozoic) & $0.22-0.35$ & 0.62 & 1.72 \\
Well cemented (Paleozoic) & $0.05-0.25$ & 0.62 & 1.95 \\
Dense, igneous, metamorphic & $<0.05$ & 1.4 & 1.6 \\
High porosity volcanic & $0.2-0.8$ & 3.5 & 1.4 \\
\hline
\end{tabular}

\section{Interpretation of geophysical data}

\subsection{Gravimetric and geomagnetic}

Integration of gravimetric and geomagnetic data with borehole data, results in the construction of maps and sections that revealed the rock characteristics and structural setting of the area.

The bulk densities for the Basement Complex around the study area vary from 2.0 to $3.06 \mathrm{~g} / \mathrm{cm}^{3}$, with mean value of $2.56 \mathrm{~g} / \mathrm{cm}^{3}$, and porosity being 3.5 to $5.9 \mathrm{~g} / \mathrm{cm}^{3}$. Matrix densities vary from 2.37 to $3.09 \mathrm{~g} / \mathrm{cm}^{3}$, with mean of $2.65 \mathrm{~g} / \mathrm{cm}$. In the Basement Complex rocks, generally low densities are caused by weathering (table 2), areas (A) and (B) referred to sectional areas.

The bulk densities for sedimentary complexes vary from 2 to 2.63 $\mathrm{g} / \mathrm{cm}^{3}$, with mean of $2.32 \mathrm{~g} / \mathrm{cm}^{3}$, and matrix densities vary from 2.38 to $2.81 \mathrm{~g} / \mathrm{cm}^{3}$, with mean of $2.6 \mathrm{~g} / \mathrm{cm}^{3}$. High densities characterize silicified sedimentary rocks of low porosity. Pelitic sediments of the recent Formations show the lowest value of the bulk densities (table 3 ). 
Table 2: Densities of Some Basement Rock Types (Source: Strojexport 1976)

\begin{tabular}{llll}
\hline Type of Rock & $\begin{array}{l}\text { Bulk Density } \\
\left(\mathrm{g} / \mathrm{cm}^{3}\right)\end{array}$ & $\begin{array}{l}\text { Matrix Densi- } \\
\text { ty }\left(\mathrm{g} / \mathrm{cm}^{3}\right)\end{array}$ & Area \\
\hline Granite, Syenite & $2.54-2.65$ & - & $\mathrm{A}$ \\
Metamorphic rocks & $2.70-2.82$ & - & $\mathrm{A}$ \\
Leucocratic pyroxene diorite & $2.66-2.70$ & - & $\mathrm{A}$ \\
Phylonitic basic rocks, diorite & $2.80-2.88$ & - & $\mathrm{A}$ \\
and quartz- tourmaline dykes & $2.19-2.37$ & - & $\mathrm{A}$ \\
Sericiti- graphite schist & 2.91 & 2.97 & $\mathrm{~B}$ \\
Amphibolite & 2.48 & 2.66 & $\mathrm{~B}$ \\
Graphite schist & 2.73 & 2.86 & $\mathrm{~B}$ \\
Biotite- muscovite schist & 2.71 & 2.73 & $\mathrm{~B}$ \\
Migmatite & $2.00-2.62$ & $2.30-2.80$ & $\mathrm{~B}$ \\
Weathered crystalline rocks & 2.54 & 2.64 & $\mathrm{~B}$ \\
Pegmatite & 2.58 & 2.61 & $\mathrm{~B}$ \\
Granite & 2.62 & 2.63 & $\mathrm{~B}$ \\
Quartz dykes & 2.63 & 2.66 & $\mathrm{~B}$ \\
Biotite gneiss & & & \\
\hline
\end{tabular}

* (A) And (B) referred to sectional areas.

Table 3: Densities of Some Sedimentary Rock Types (Source: Strojexport 1976)

\begin{tabular}{llll}
\hline Type of Rock & $\begin{array}{l}\text { Bulk Density } \\
\left(\mathrm{g} / \mathrm{cm}^{3}\right)\end{array}$ & $\begin{array}{l}\text { Matrix Densi- } \\
\mathrm{ty}\left(\mathrm{g} / \mathrm{cm}^{3}\right)\end{array}$ & Area \\
\hline $\begin{array}{l}\text { Slightly cemented mudstone } \\
\text { and siltstone }\end{array}$ & $2.00-2.18$ & - & $\mathrm{A}$ \\
$\begin{array}{l}\text { Slightly cemented sandstones } \\
\text { and conglomerates }\end{array}$ & $2.26-2.34$ & - & $\mathrm{A}$ \\
$\begin{array}{l}\text { Silicified sandstones and } \\
\text { conglomerates }\end{array}$ & $2.42-2.63$ & 2.72 & $\mathrm{~A}$ \\
$\begin{array}{l}\text { Sands and sandstones } \\
\text { Mudstones and siltstones }\end{array}$ & 1.76 & 2.52 & $\mathrm{~B}$ \\
Clays & 2.06 & 2.62 & $\mathrm{~B}$ \\
& $1.52-2.12$ & - & $\mathrm{B}$ \\
\hline
\end{tabular}

Laterites show bulk densities of 2.18 to $3.21 \mathrm{~g} / \mathrm{cm}^{3}$. Volcanic rocks from locations outside the study area (Basalts, Tuffs and agglomerates) show mean bulk density of $2.48 \mathrm{~g} / \mathrm{cm}^{3}$ and mean matrix density of $2.62 \mathrm{~g} / \mathrm{cm}^{3}$.

The analysis of densities and their variations has resulted in a conclusion that the reduction density of $2.67 \mathrm{~g} / \mathrm{cm}^{3}$ would be the most suitable one for the Bouguer reduction of the gravity to obtain the Bouguer gravity.

Bouguer gravity map (Fig. 4) clearly defined trends and depths of structural elements which dissect the study area. The gravity values show intense gradient and range from -22.5 in the north to -75 mgal in the south, indicating increasing depth and thickness northsouth direction.

Gravity values show elevations and depressions which can be related to structural or lithological units. The elevations may be due to elevated blocks or they may be due to injected intrusions that not come out to the surface or due to silicification along fault zones. Depressions, elevations and effect of lithology also manifest in gravity sections (Figs. 5). The ambiguity in gravity data is partly eliminated by borehole logs and other geophysical measurements (Figs. 6).

Measurements of the " $\mathrm{z}$ " component of the geomagnetic field show anomalous high readings, which interpreted as the cause of basic rock complexes or due to effect of ferruginous sandstones and conglomerates in the Nubian Series and laterites. Mineralization along fault zones also may cause anomalous values in magnetic responses. (Figs. 7) show combinations of gravity and magnetic measurements and inconsistent responses. Nevertheless these combinations gave more precision to geological interpretation of gravity measurements.

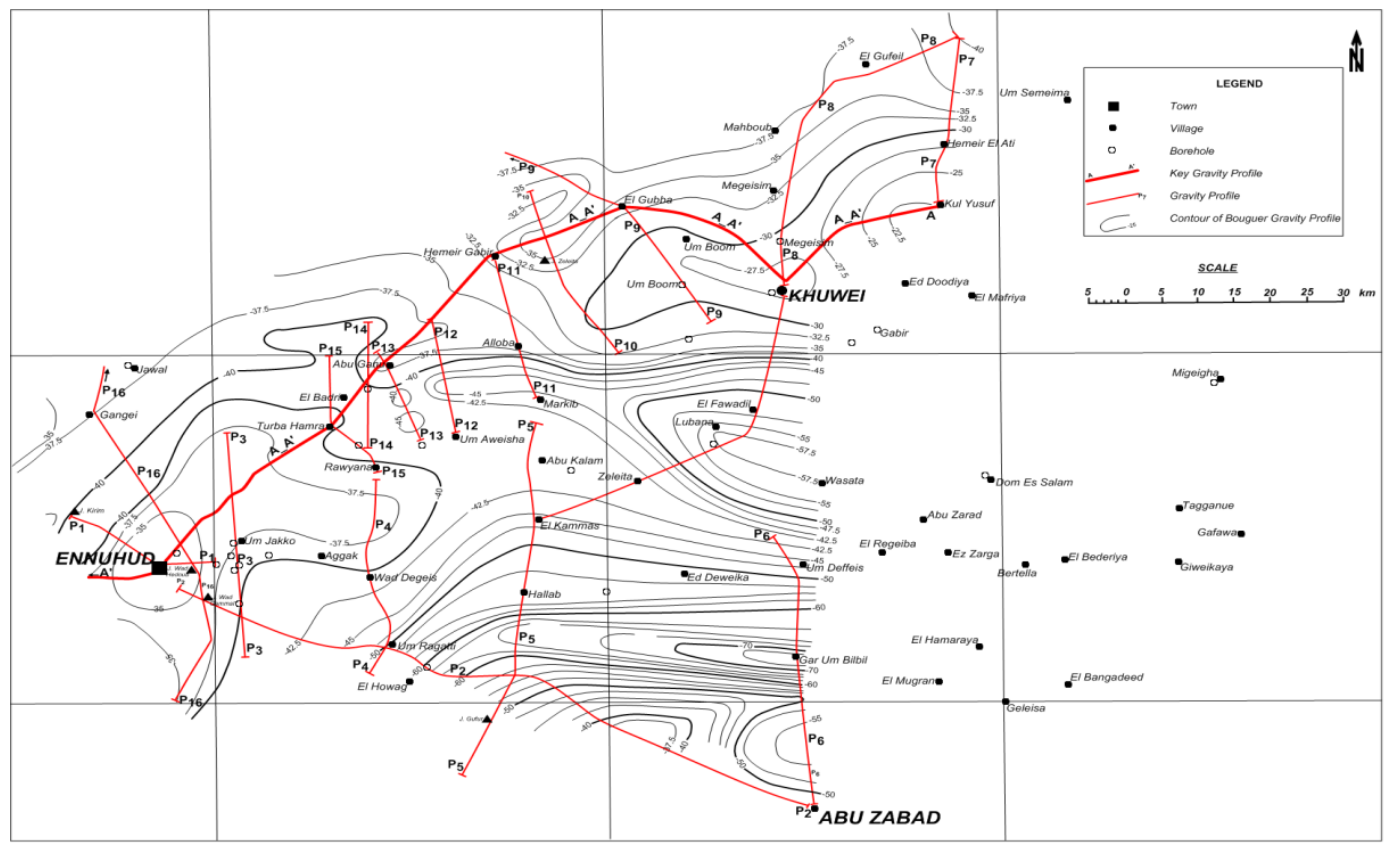

Fig. 4: Bouguer Gravity Contours (Based On Strojexport 1975, Plate 13, Area B, En Nuhud-El Khuwei). 


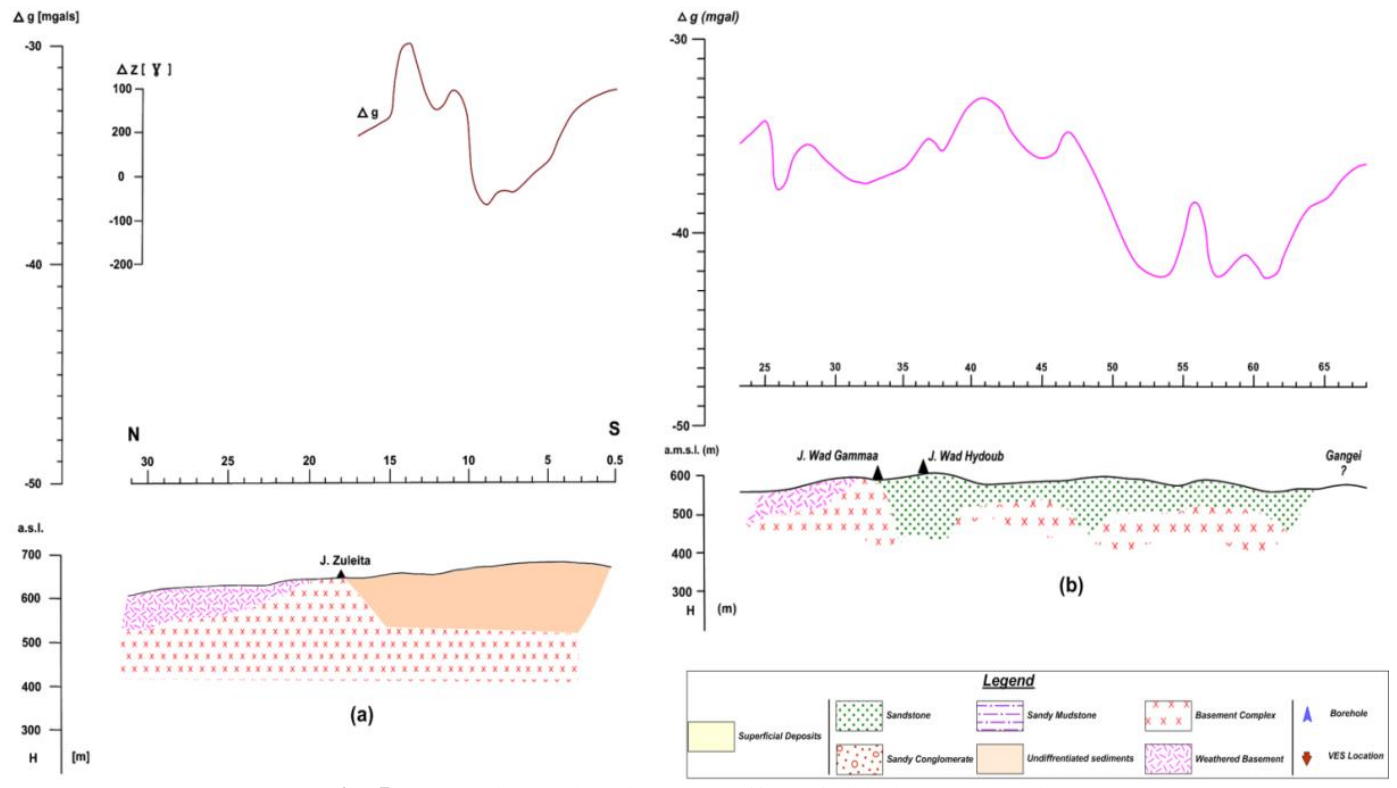

Fig. 5: Depressions, Elevations and Effect of Lithology on Gravity.

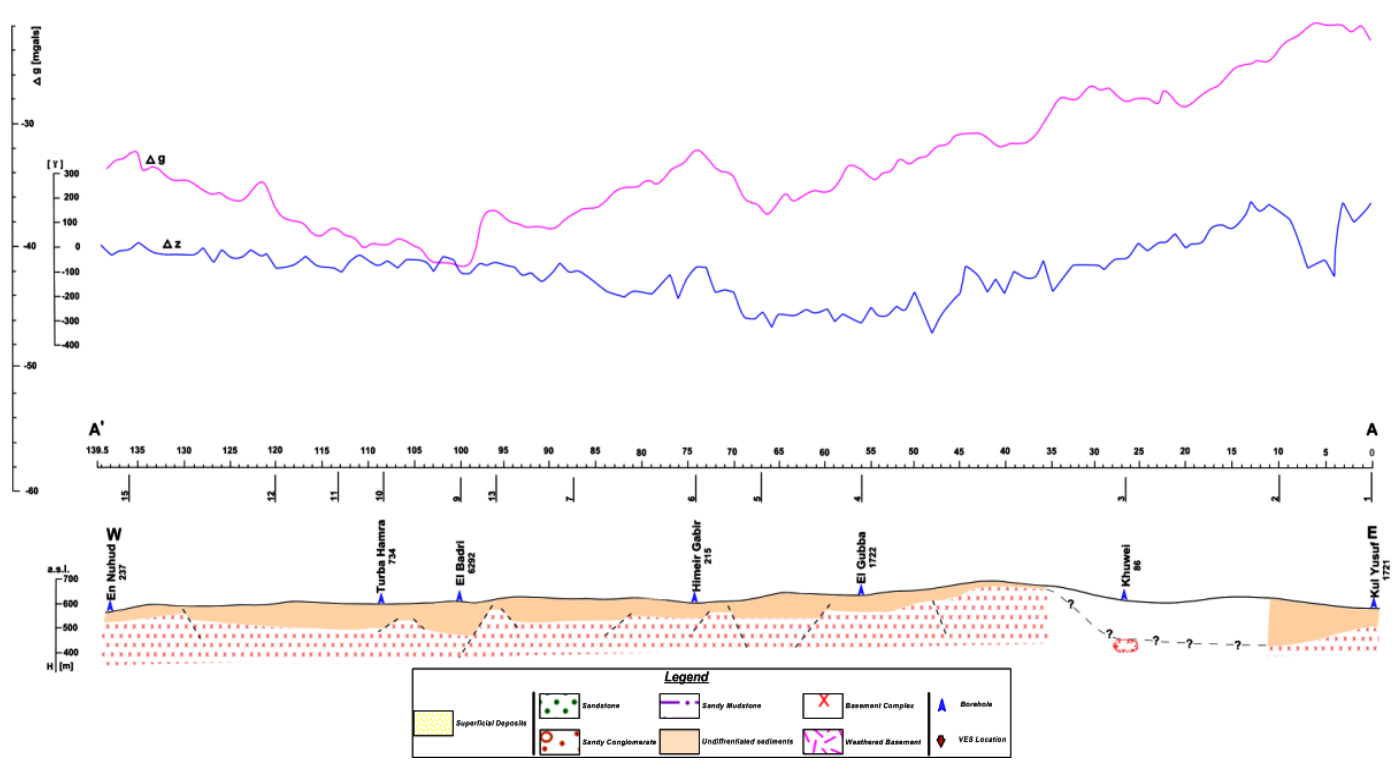

Fig. 6: Elimination of Ambiguity on Gravity by other Measurements.

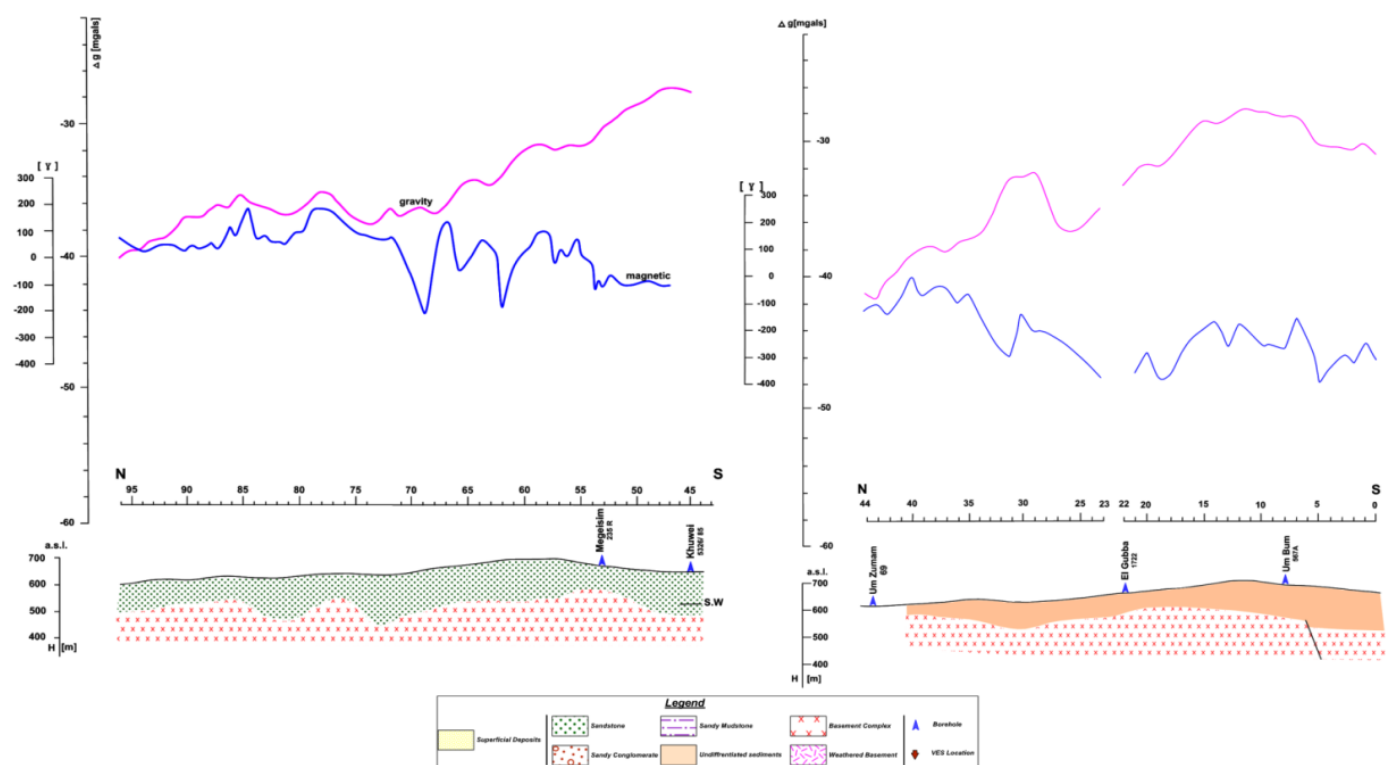

Fig. 7: Combination of Gravity and Magnetic Measurements. 


\subsection{Geoelectric}

Analysis of resistivity data are presented as apparent resistivity and thickness of layers, depth to Basement Complex and types of VES curves (table 4). The dominant type curves are 7-layer $(31 \%)$, 5-layer $(27.6 \%)$ and 4-layer $(22.4 \%)$. The 4-layer and 3layer types usually corresponds to shallow Basement areas. Based on table (4) and equations (4) and (5) formation factor " $F "$ and porosity " $\phi$ " were calculated. From the resistivity data, values of " $\mathrm{R}_{\mathrm{f}}$ " is determined, and then values of " $\mathrm{R}_{\mathrm{w}}$ ", " $F$ " and " $\phi$ " were calculated for some locations in the study area. The values ob- tained are: (52- 422 ohm.m) for " $\mathrm{R}_{\mathrm{f}}$ ", (26.39- 65.36 ohm.m) for "R $\mathrm{R}_{\mathrm{w}}$, (1.73- 11.60) for "F", and (18- 50.9\%) for " $\phi "$ (table 5). Qualitative interpretation of resistivity data indicate three stratigraphic units; the Superficial Deposits, Nubian Sandstone Formation, and the Basement Complex.

The Superficial Deposits at the surface of the sequence notes a wide range of resistivity values as $6.26-18288 \mathrm{ohm} . \mathrm{m}$, which can be justified as due to variability in mineralogical composition, texture, or moisture content at the measuring time, with remarking that most resistivity values indicate sandy materials.

Table 4: Results of Resistivity Interpretation [Thickness (H) and Depth in Meters, Resistivity (ঐ) in Ohm. M].

\begin{tabular}{|c|c|c|c|c|c|c|c|c|c|c|c|c|c|c|c|c|c|c|}
\hline $\begin{array}{l}\text { VE } \\
\text { S } \\
\text { No }\end{array}$ & $\begin{array}{l}\text { EAST- } \\
\text { ING }\end{array}$ & $\begin{array}{l}\text { NATES } \\
\text { NORTH- } \\
\text { ING }\end{array}$ & $\begin{array}{l}\text { Elev } \\
\text { a- } \\
\text { tion }\end{array}$ & $\begin{array}{l}\text { VES } \\
\text { TYPE }\end{array}$ & $\begin{array}{l}\text { oll } 1 \\
\text { ohm. } \\
\text { m }\end{array}$ & $\begin{array}{l}\mathrm{h}_{1} \\
(\mathrm{~m} \\
)\end{array}$ & $\begin{array}{l}\text { तi2 } \\
\text { ohm. } \\
\text { m }\end{array}$ & $\begin{array}{l}\mathrm{h}_{2} \\
(\mathrm{~m})\end{array}$ & $\begin{array}{l}\text { ohm. } \\
\text { m }\end{array}$ & $\begin{array}{l}\mathrm{h}_{3} \\
(\mathrm{~m})\end{array}$ & $\begin{array}{l}\text { तif } \\
\text { ohm. } \\
\text { m }\end{array}$ & $\begin{array}{l}\mathrm{h}_{4} \\
(\mathrm{~m} \\
)\end{array}$ & $\begin{array}{l}\text { an5 } \\
\text { ohm. } \\
\text { m }\end{array}$ & $\begin{array}{l}\mathrm{h}_{5} \\
(\mathrm{~m} \\
)\end{array}$ & $\begin{array}{l}\text { ei6 } \\
\text { ohm. } \\
\text { m }\end{array}$ & $\begin{array}{l}\mathrm{h}_{6} \\
(\mathrm{~m})\end{array}$ & $\begin{array}{l}\text { ait } \\
\text { ohm. } \\
\text { m }\end{array}$ & $\begin{array}{l}\text { Dept } \\
\text { h to } \\
\text { B.C. } \\
\text { (m) }\end{array}$ \\
\hline 1 & 29.20 & 13.10 & 635 & HKH & 1108 & $\begin{array}{l}0.7 \\
5\end{array}$ & $\begin{array}{l}62.8 \\
9\end{array}$ & $\begin{array}{l}7.4 \\
3\end{array}$ & $\begin{array}{l}524 . \\
3\end{array}$ & $\begin{array}{l}91 . \\
34\end{array}$ & $\begin{array}{l}84.2 \\
0\end{array}$ & $\begin{array}{l}37 . \\
8\end{array}$ & 139 & - & - & - & - & 13. \\
\hline 2 & 29.20 & 13.10 & 633 & $\begin{array}{l}\text { HKQ } \\
\mathrm{H}\end{array}$ & 1137 & $\begin{array}{l}2.5 \\
5\end{array}$ & 69.1 & $\begin{array}{l}37 . \\
33\end{array}$ & 1614 & $\begin{array}{l}71 . \\
88\end{array}$ & 200 & 34 & 50 & 8.0 & $\begin{array}{l}176 . \\
3\end{array}$ & - & - & 153 \\
\hline 3 & 29.21 & 13.10 & 634 & $\begin{array}{l}\text { QHK } \\
\text { HA }\end{array}$ & $\begin{array}{l}81.8 \\
4\end{array}$ & $\begin{array}{l}2.4 \\
8\end{array}$ & $\begin{array}{l}30.7 \\
7\end{array}$ & $\begin{array}{l}13 . \\
39\end{array}$ & $\begin{array}{l}10.4 \\
3\end{array}$ & $\begin{array}{l}4.1 \\
2\end{array}$ & $\begin{array}{l}942 . \\
8\end{array}$ & $\begin{array}{l}16 . \\
8\end{array}$ & 7.10 & $\begin{array}{l}17 . \\
7\end{array}$ & $\begin{array}{l}191 . \\
3\end{array}$ & 79 & 2420 & $\begin{array}{l}133 . \\
45\end{array}$ \\
\hline 4 & - & - & - & $\begin{array}{l}\text { QHK } \\
\text { HK }\end{array}$ & $\begin{array}{l}722 . \\
5\end{array}$ & $\begin{array}{l}0.3 \\
2\end{array}$ & $\begin{array}{l}71.7 \\
9\end{array}$ & $\begin{array}{l}5.4 \\
41\end{array}$ & $\begin{array}{l}27.2 \\
8\end{array}$ & $\begin{array}{l}1.5 \\
29\end{array}$ & 1534 & $\begin{array}{l}2.9 \\
0\end{array}$ & $\begin{array}{l}202 . \\
3\end{array}$ & $\begin{array}{l}0.2 \\
9\end{array}$ & $\begin{array}{l}540 . \\
6\end{array}$ & $\begin{array}{l}109 \\
.6\end{array}$ & 160 & $\begin{array}{l}>12 \\
0\end{array}$ \\
\hline 5 & 29.10 & 13.00 & 657 & $\mathrm{AKH}$ & 100 & $\begin{array}{l}0.3 \\
8\end{array}$ & 1165 & $\begin{array}{l}2.1 \\
39\end{array}$ & 8182 & $\begin{array}{l}20 . \\
28\end{array}$ & $\begin{array}{l}180 . \\
4\end{array}$ & $\begin{array}{l}13 \\
5\end{array}$ & 2063 & - & - & - & - & 158 \\
\hline 6 & 29.10 & 13.00 & 668 & HKQ & $\begin{array}{l}610 . \\
5\end{array}$ & $\begin{array}{l}0.9 \\
9\end{array}$ & 7.24 & $\begin{array}{l}2.2 \\
66\end{array}$ & 1869 & $\begin{array}{l}15 . \\
92\end{array}$ & 1840 & $\begin{array}{l}13 \\
3\end{array}$ & $\begin{array}{l}97.5 \\
9\end{array}$ & - & - & - & - & $\begin{array}{l}152 . \\
4\end{array}$ \\
\hline 7 & 29.10 & 13.00 & 662 & HKQ & $\begin{array}{l}508 . \\
1\end{array}$ & $\begin{array}{l}1.5 \\
3\end{array}$ & 9.67 & $\begin{array}{l}1.7 \\
40\end{array}$ & 1741 & $\begin{array}{l}15 . \\
51\end{array}$ & 1286 & $\begin{array}{l}12 \\
7\end{array}$ & $\begin{array}{l}96.4 \\
6\end{array}$ & - & - & - & - & $\begin{array}{l}145 . \\
31\end{array}$ \\
\hline 9 & 29.30 & 12.90 & 628 & HK & 3998 & $\begin{array}{l}2.3 \\
2\end{array}$ & $\begin{array}{l}70.0 \\
3\end{array}$ & $\begin{array}{l}15 . \\
78\end{array}$ & 1145 & $\begin{array}{l}118 \\
.8\end{array}$ & 78.0 & - & - & - & - & - & - & 135 \\
\hline 10 & 28.90 & 13.10 & 614 & $\mathrm{QH}$ & 968 & $\begin{array}{l}4.9 \\
3\end{array}$ & $\begin{array}{l}45.7 \\
5\end{array}$ & $\begin{array}{l}20 . \\
30\end{array}$ & 3.14 & $\begin{array}{l}18 . \\
95\end{array}$ & $\begin{array}{l}255 \\
6\end{array}$ & - & - & - & - & - & - & 44 \\
\hline 11 & 28.80 & 13.00 & 658 & HAK & 1180 & $\begin{array}{l}1.4 \\
3\end{array}$ & $\begin{array}{l}17.8 \\
9\end{array}$ & $\begin{array}{l}0.7 \\
2\end{array}$ & $\begin{array}{l}381 . \\
4\end{array}$ & $\begin{array}{l}8.6 \\
14\end{array}$ & 437 & $\begin{array}{l}69 . \\
5\end{array}$ & $\begin{array}{l}164 . \\
3\end{array}$ & - & - & - & - & 122 \\
\hline 12 & 28.80 & 13.00 & 646 & HAK & $\begin{array}{l}605 . \\
6\end{array}$ & $\begin{array}{l}2.1 \\
9\end{array}$ & $\begin{array}{l}43.7 \\
7\end{array}$ & $\begin{array}{l}24 . \\
26\end{array}$ & $\begin{array}{l}222 . \\
8\end{array}$ & $\begin{array}{l}24 . \\
01\end{array}$ & $\begin{array}{l}479 . \\
4\end{array}$ & $\begin{array}{l}14 . \\
1\end{array}$ & $\begin{array}{l}149 . \\
6\end{array}$ & - & - & - & - & 122 \\
\hline 13 & 28.84 & 13.00 & 634 & HAK & 2320 & $\begin{array}{l}3.6 \\
5\end{array}$ & $\begin{array}{l}72.4 \\
5\end{array}$ & $\begin{array}{l}43 . \\
1\end{array}$ & $\begin{array}{l}908 . \\
4\end{array}$ & $\begin{array}{l}17 . \\
94\end{array}$ & 3082 & $\begin{array}{l}59 . \\
6\end{array}$ & $\begin{array}{l}276 . \\
3\end{array}$ & - & - & - & - & 125 \\
\hline 14 & 28.87 & 12.92 & 622 & $\begin{array}{l}\text { QHK } \\
\text { HK }\end{array}$ & 2224 & $\begin{array}{l}1.2 \\
4\end{array}$ & $\begin{array}{l}188 . \\
9\end{array}$ & $\begin{array}{l}4.2 \\
3\end{array}$ & 9.69 & $\begin{array}{l}3.6 \\
3\end{array}$ & $\begin{array}{l}640 . \\
8\end{array}$ & $\begin{array}{l}10 . \\
4\end{array}$ & $\begin{array}{l}25.2 \\
5\end{array}$ & $\begin{array}{l}4.9 \\
7\end{array}$ & $\begin{array}{l}684 . \\
9\end{array}$ & $\begin{array}{l}69 . \\
09\end{array}$ & $\begin{array}{l}102 . \\
8\end{array}$ & $\begin{array}{l}>23 \\
0\end{array}$ \\
\hline 15 & 28.42 & 12.54 & 627 & $\begin{array}{l}\text { QHK } \\
\text { QH }\end{array}$ & 4749 & $\begin{array}{l}1.0 \\
4\end{array}$ & $\begin{array}{l}187 . \\
6\end{array}$ & $\begin{array}{l}6.2 \\
51\end{array}$ & 9.80 & $\begin{array}{l}2.4 \\
88\end{array}$ & 2033 & $\begin{array}{l}4.1 \\
0\end{array}$ & 201 & $\begin{array}{l}0.5 \\
2\end{array}$ & $\begin{array}{l}56.2 \\
9\end{array}$ & $\begin{array}{l}119 \\
.9\end{array}$ & 3322 & $\begin{array}{l}100 . \\
6\end{array}$ \\
\hline 16 & 28.60 & 12.90 & 613 & $\begin{array}{l}\text { QQQ } \\
\mathrm{H}\end{array}$ & 4567 & $\begin{array}{l}0.8 \\
1\end{array}$ & 1354 & $\begin{array}{l}3.7 \\
1\end{array}$ & 176 & $\begin{array}{l}18 . \\
7\end{array}$ & 34.3 & $\begin{array}{l}81 . \\
9\end{array}$ & 2.69 & $\begin{array}{l}28 . \\
9\end{array}$ & 6235 & - & - & 134 \\
\hline 18 & 28.40 & 13.00 & 559 & $\begin{array}{l}\text { HKQ } \\
\mathrm{H}\end{array}$ & $\begin{array}{l}149 . \\
8\end{array}$ & $\begin{array}{l}0.9 \\
0\end{array}$ & $\begin{array}{l}80.7 \\
5\end{array}$ & $\begin{array}{l}10 . \\
29\end{array}$ & $\begin{array}{l}904 . \\
6\end{array}$ & $\begin{array}{l}4.9 \\
0\end{array}$ & 6.64 & $\begin{array}{l}53 . \\
4\end{array}$ & $\begin{array}{l}17.6 \\
9\end{array}$ & $\begin{array}{l}12 \\
6\end{array}$ & $\begin{array}{l}893 . \\
2\end{array}$ & - & - & 195 \\
\hline 19 & 28.40 & 13.00 & 559 & HKH & 1046 & $\begin{array}{l}1.5 \\
3\end{array}$ & $\begin{array}{l}80.0 \\
7\end{array}$ & $\begin{array}{l}1.0 \\
1\end{array}$ & $\begin{array}{l}493 . \\
2\end{array}$ & $\begin{array}{l}26 . \\
96\end{array}$ & $\begin{array}{l}30.4 \\
1\end{array}$ & $\begin{array}{l}46 . \\
5\end{array}$ & $\begin{array}{l}358 . \\
7\end{array}$ & - & - & - & - & 75 \\
\hline 20 & 28.40 & 13.00 & 561 & HKH & $\begin{array}{l}262 . \\
6\end{array}$ & $\begin{array}{l}2.2 \\
4\end{array}$ & $\begin{array}{l}68.7 \\
5\end{array}$ & $\begin{array}{l}2.9 \\
3\end{array}$ & $\begin{array}{l}793 . \\
3\end{array}$ & $\begin{array}{l}7.4 \\
0\end{array}$ & 8.08 & $\begin{array}{l}87 . \\
4\end{array}$ & $\begin{array}{l}569 . \\
5\end{array}$ & - & - & - & - & 100 \\
\hline 21 & 28.40 & 13.00 & 559 & $\begin{array}{l}\text { HKQ } \\
\mathrm{H}\end{array}$ & $\begin{array}{l}144 . \\
3\end{array}$ & $\begin{array}{l}0.1 \\
0\end{array}$ & $\begin{array}{l}78.9 \\
6\end{array}$ & $\begin{array}{l}9.7 \\
7\end{array}$ & $\begin{array}{l}945 . \\
4\end{array}$ & $\begin{array}{l}4.6 \\
2\end{array}$ & 9.02 & $\begin{array}{l}93 . \\
7\end{array}$ & 20.0 & $\begin{array}{l}96 . \\
7\end{array}$ & 1003 & - & - & 205 \\
\hline 22 & 28.40 & 12.80 & 613 & $\mathrm{QH}$ & 455 & $\begin{array}{l}0.5 \\
9\end{array}$ & $\begin{array}{l}33.9 \\
7\end{array}$ & $\begin{array}{l}14 . \\
57\end{array}$ & 8.66 & $\begin{array}{l}45 . \\
06\end{array}$ & $\begin{array}{l}306 . \\
8\end{array}$ & - & - & - & - & - & - & 60 \\
\hline 23 & 28.50 & 12.80 & 594 & QQH & $\begin{array}{l}251 . \\
2\end{array}$ & $\begin{array}{l}1.7 \\
9\end{array}$ & $\begin{array}{l}66.5 \\
1\end{array}$ & $\begin{array}{l}8.5 \\
1\end{array}$ & $\begin{array}{l}94.8 \\
8\end{array}$ & $\begin{array}{l}5.0 \\
6\end{array}$ & $\begin{array}{l}39.8 \\
4\end{array}$ & $\begin{array}{l}55 . \\
8\end{array}$ & $\begin{array}{l}237 . \\
6\end{array}$ & - & - & - & - & $\begin{array}{l}71.6 \\
8\end{array}$ \\
\hline 24 & 28.50 & 12.70 & 624 & $\begin{array}{l}\text { HKH } \\
\text { KQ }\end{array}$ & 2304 & $\begin{array}{l}1.5 \\
0\end{array}$ & $\begin{array}{l}67.9 \\
5\end{array}$ & $\begin{array}{l}11 . \\
36\end{array}$ & 1374 & $\begin{array}{l}11 . \\
68\end{array}$ & $\begin{array}{l}66.8 \\
1\end{array}$ & $\begin{array}{l}19 . \\
0\end{array}$ & 2354 & $\begin{array}{l}68 . \\
4\end{array}$ & $\begin{array}{l}405 . \\
8\end{array}$ & $\begin{array}{l}46 . \\
12\end{array}$ & $\begin{array}{l}42.5 \\
2\end{array}$ & $\begin{array}{l}>15 \\
8\end{array}$ \\
\hline 25 & 28.50 & 12.70 & 624 & $\begin{array}{l}\text { HKH } \\
\text { KQ }\end{array}$ & 2906 & $\begin{array}{l}3.0 \\
2\end{array}$ & $\begin{array}{l}65.1 \\
9\end{array}$ & $\begin{array}{l}10 . \\
83\end{array}$ & 5611 & $\begin{array}{l}12 . \\
44\end{array}$ & $\begin{array}{l}140 . \\
3\end{array}$ & $\begin{array}{l}55 . \\
1\end{array}$ & 800 & $\begin{array}{l}30 . \\
6\end{array}$ & $\begin{array}{l}398 . \\
4\end{array}$ & 55 & $\begin{array}{l}69.7 \\
6\end{array}$ & $\begin{array}{l}>16 \\
6\end{array}$ \\
\hline 26 & 28.50 & 12.70 & 624 & $\begin{array}{l}\text { HKH } \\
\text { KQ }\end{array}$ & 2935 & $\begin{array}{l}2.5 \\
6\end{array}$ & $\begin{array}{l}34.6 \\
3\end{array}$ & $\begin{array}{l}8.3 \\
3\end{array}$ & 1676 & $\begin{array}{l}23 . \\
05\end{array}$ & $\begin{array}{l}69.6 \\
5\end{array}$ & $\begin{array}{l}19 . \\
4\end{array}$ & $\begin{array}{l}1843 \\
.2\end{array}$ & $\begin{array}{l}58 . \\
6\end{array}$ & $\begin{array}{l}393 . \\
2\end{array}$ & $\begin{array}{l}32 . \\
06\end{array}$ & $\begin{array}{l}41.0 \\
5\end{array}$ & $\begin{array}{l}>14 \\
4\end{array}$ \\
\hline 27 & 28.80 & 12.60 & 620 & $\begin{array}{l}\text { KHA } \\
\text { A }\end{array}$ & 6.71 & $\begin{array}{l}0.0 \\
8\end{array}$ & $\begin{array}{l}963 . \\
1\end{array}$ & $\begin{array}{l}10 . \\
4\end{array}$ & $\begin{array}{l}442 . \\
2\end{array}$ & 136 & 3128 & $\begin{array}{l}89 . \\
6\end{array}$ & $\begin{array}{l}1674 \\
0\end{array}$ & $\begin{array}{l}63 . \\
7\end{array}$ & $\infty$ & - & - & 279 \\
\hline 28 & 28.71 & 12.55 & 636 & $\begin{array}{l}\text { QHK } \\
\text { QH }\end{array}$ & $\begin{array}{l}134 . \\
1\end{array}$ & $\begin{array}{l}1.5 \\
5\end{array}$ & $\begin{array}{l}47.1 \\
7\end{array}$ & $\begin{array}{l}5.1 \\
2\end{array}$ & $\begin{array}{l}42.2 \\
0\end{array}$ & $\begin{array}{l}0.8 \\
76\end{array}$ & 1827 & $\begin{array}{l}6.3 \\
4\end{array}$ & $\begin{array}{l}184 . \\
5\end{array}$ & $\begin{array}{l}0.6 \\
2\end{array}$ & $\begin{array}{l}31.0 \\
2\end{array}$ & $\begin{array}{l}185 \\
.4\end{array}$ & $\begin{array}{l}383 . \\
3\end{array}$ & $\begin{array}{l}>20 \\
0\end{array}$ \\
\hline 29 & 28.90 & 12.70 & 609 & $\begin{array}{l}\text { QHK } \\
\text { HK }\end{array}$ & 2347 & $\begin{array}{l}0.4 \\
3\end{array}$ & $\begin{array}{l}65.1 \\
6\end{array}$ & $\begin{array}{l}17 . \\
78\end{array}$ & $\begin{array}{l}12.9 \\
9\end{array}$ & $\begin{array}{l}3.3 \\
2\end{array}$ & $\begin{array}{l}740 . \\
6\end{array}$ & $\begin{array}{l}13 . \\
3\end{array}$ & 9.47 & $\begin{array}{l}11 . \\
9\end{array}$ & $\begin{array}{l}412 . \\
6\end{array}$ & $\begin{array}{l}60 . \\
67\end{array}$ & $\begin{array}{l}81.2 \\
9\end{array}$ & $\begin{array}{l}>23 \\
0\end{array}$ \\
\hline 30 & 28.90 & 12.70 & 609 & $\begin{array}{l}\text { QHK } \\
\text { HK }\end{array}$ & $\begin{array}{l}463 . \\
8\end{array}$ & $\begin{array}{l}1.8 \\
9\end{array}$ & $\begin{array}{l}69.7 \\
8\end{array}$ & $\begin{array}{l}26 . \\
12\end{array}$ & $\begin{array}{l}11.8 \\
7\end{array}$ & $\begin{array}{l}3.7 \\
6\end{array}$ & $\begin{array}{l}561 . \\
1\end{array}$ & $\begin{array}{l}10 . \\
3\end{array}$ & 5.63 & $\begin{array}{l}19 . \\
8\end{array}$ & $\begin{array}{l}371 . \\
2\end{array}$ & $\begin{array}{l}57 . \\
51\end{array}$ & 164 & $\begin{array}{l}>23 \\
0\end{array}$ \\
\hline
\end{tabular}




\begin{tabular}{|c|c|c|c|c|c|c|c|c|c|c|c|c|c|c|c|c|c|c|}
\hline 31 & 29.91 & 12.75 & 614 & $\begin{array}{l}\text { QHK } \\
\text { HK }\end{array}$ & $\begin{array}{l}658 . \\
4\end{array}$ & $\begin{array}{l}3.3 \\
8\end{array}$ & $\begin{array}{l}78.2 \\
7\end{array}$ & $\begin{array}{l}10 . \\
74\end{array}$ & 7.00 & $\begin{array}{l}5.3 \\
7\end{array}$ & 2776 & $\begin{array}{l}29 . \\
6\end{array}$ & $\begin{array}{l}97.7 \\
7\end{array}$ & $\begin{array}{l}1.2 \\
8\end{array}$ & 2640 & $\begin{array}{l}74 . \\
55\end{array}$ & $\begin{array}{l}62.4 \\
8\end{array}$ & $\begin{array}{l}>23 \\
0\end{array}$ \\
\hline 32 & 29.24 & 12.65 & 599 & $\begin{array}{l}\text { QHK } \\
\text { HK }\end{array}$ & 3011 & $\begin{array}{l}1.2 \\
5\end{array}$ & $\begin{array}{l}149 . \\
1\end{array}$ & $\begin{array}{l}4.8 \\
1\end{array}$ & 9.32 & $\begin{array}{l}6.6 \\
1\end{array}$ & 1439 & $\begin{array}{l}5.0 \\
3\end{array}$ & $\begin{array}{l}203 . \\
5\end{array}$ & $\begin{array}{l}0.5 \\
1\end{array}$ & $\begin{array}{l}462 . \\
2\end{array}$ & $\begin{array}{l}114 \\
.9\end{array}$ & $\begin{array}{l}41.8 \\
0\end{array}$ & $\begin{array}{l}>20 \\
0\end{array}$ \\
\hline 33 & 29.20 & 12.50 & 595 & $\begin{array}{l}\text { HKH } \\
\text { KQ }\end{array}$ & 2198 & $\begin{array}{l}1.4 \\
0\end{array}$ & $\begin{array}{l}36.0 \\
2\end{array}$ & $\begin{array}{l}22 . \\
66\end{array}$ & 1006 & $\begin{array}{l}14 . \\
31\end{array}$ & 5.69 & $\begin{array}{l}74 . \\
7\end{array}$ & $\begin{array}{l}145 . \\
9\end{array}$ & $\begin{array}{l}15 \\
3\end{array}$ & $\begin{array}{l}40.7 \\
6\end{array}$ & $\begin{array}{l}76 . \\
51\end{array}$ & 17.0 & $\begin{array}{l}>34 \\
2\end{array}$ \\
\hline 34 & 29.65 & 12.81 & 656 & $\begin{array}{l}\text { QHK } \\
\text { HK }\end{array}$ & 3763 & $\begin{array}{l}0.2 \\
6\end{array}$ & $\begin{array}{l}103 . \\
8\end{array}$ & $\begin{array}{l}10 . \\
50\end{array}$ & $\begin{array}{l}38.7 \\
1\end{array}$ & $\begin{array}{l}0.6 \\
03\end{array}$ & 5289 & $\begin{array}{l}6.7 \\
2\end{array}$ & $\begin{array}{l}189 . \\
4\end{array}$ & $\begin{array}{l}0.3 \\
4\end{array}$ & $\begin{array}{l}953 . \\
8\end{array}$ & $\begin{array}{l}117 \\
.6\end{array}$ & $\begin{array}{l}32.1 \\
4\end{array}$ & $\begin{array}{l}>13 \\
6\end{array}$ \\
\hline 35 & 29.53 & 12.72 & 614 & HKH & $\begin{array}{l}71.3 \\
8\end{array}$ & $\begin{array}{l}2.0 \\
0\end{array}$ & $\begin{array}{l}22.5 \\
2\end{array}$ & $\begin{array}{l}26 . \\
90\end{array}$ & $\begin{array}{l}400 . \\
7\end{array}$ & $\begin{array}{l}4.2 \\
6\end{array}$ & 5.86 & $\begin{array}{l}5.8 \\
6\end{array}$ & $\begin{array}{l}22.2 \\
5\end{array}$ & $\begin{array}{l}58 . \\
6\end{array}$ & - & - & - & $\begin{array}{l}>20 \\
0\end{array}$ \\
\hline 36 & 29.56 & 12.75 & 624 & $\begin{array}{l}\text { QHA } \\
\mathrm{KH}\end{array}$ & $\begin{array}{l}455 . \\
1\end{array}$ & $\begin{array}{l}0.4 \\
2\end{array}$ & $\begin{array}{l}145 . \\
1\end{array}$ & $\begin{array}{l}1.5 \\
4\end{array}$ & $\begin{array}{l}18.1 \\
7\end{array}$ & $\begin{array}{l}5.9 \\
3\end{array}$ & $\begin{array}{l}20.7 \\
4\end{array}$ & $\begin{array}{l}5.2 \\
6\end{array}$ & $\begin{array}{l}3558 \\
.7\end{array}$ & $\begin{array}{l}31 . \\
6\end{array}$ & $\begin{array}{l}40.5 \\
0\end{array}$ & $\begin{array}{l}162 \\
.8\end{array}$ & 417 & $\begin{array}{l}>20 \\
0\end{array}$ \\
\hline 37 & 29.55 & 12.75 & 624 & $\begin{array}{l}\text { HAA } \\
\text { AK }\end{array}$ & $\begin{array}{l}316 . \\
6\end{array}$ & $\begin{array}{l}0.3 \\
3\end{array}$ & $\begin{array}{l}23.1 \\
3\end{array}$ & $\begin{array}{l}1.6 \\
3\end{array}$ & $\begin{array}{l}32.8 \\
6\end{array}$ & $\begin{array}{l}0.6 \\
21\end{array}$ & $\begin{array}{l}47.2 \\
2\end{array}$ & $\begin{array}{l}17 . \\
1\end{array}$ & $\begin{array}{l}508 . \\
8\end{array}$ & $\begin{array}{l}44 . \\
9\end{array}$ & $\begin{array}{l}659 . \\
8\end{array}$ & $\begin{array}{l}126 \\
.6\end{array}$ & $\begin{array}{l}83.3 \\
8\end{array}$ & $\begin{array}{l}>20 \\
0\end{array}$ \\
\hline 38 & 29.35 & 13.03 & 615 & $\begin{array}{l}\text { QHK } \\
\text { HA }\end{array}$ & $\begin{array}{l}1828 \\
8\end{array}$ & $\begin{array}{l}0.3 \\
7\end{array}$ & 3456 & $\begin{array}{l}1.2 \\
95\end{array}$ & $\begin{array}{l}54.1 \\
0\end{array}$ & $\begin{array}{l}0.4 \\
55\end{array}$ & $\begin{array}{l}358 . \\
8\end{array}$ & $\begin{array}{l}1.9 \\
6\end{array}$ & $\begin{array}{l}23.5 \\
1\end{array}$ & $\begin{array}{l}3.1 \\
5\end{array}$ & $\begin{array}{l}111 . \\
3\end{array}$ & $\begin{array}{l}112 \\
.8\end{array}$ & 1639 & 120 \\
\hline 39 & 29.36 & 13.03 & 617 & $\begin{array}{l}\text { AHK } \\
\text { HK }\end{array}$ & 4418 & $\begin{array}{l}1.9 \\
1\end{array}$ & 1120 & $\begin{array}{l}8.2 \\
00\end{array}$ & $\begin{array}{l}20.4 \\
7\end{array}$ & $\begin{array}{l}4.7 \\
70\end{array}$ & $\begin{array}{l}225 . \\
3\end{array}$ & $\begin{array}{l}8.5 \\
1\end{array}$ & $\begin{array}{l}14.1 \\
1\end{array}$ & $\begin{array}{l}17 . \\
9\end{array}$ & $\begin{array}{l}352 . \\
7\end{array}$ & $\begin{array}{l}95 . \\
4\end{array}$ & $\begin{array}{l}74.9 \\
5\end{array}$ & $\begin{array}{l}136 . \\
38\end{array}$ \\
\hline 41 & 29.40 & 12.40 & 500 & QHA & 1870 & $\begin{array}{l}0.3 \\
8\end{array}$ & $\begin{array}{l}88.6 \\
5\end{array}$ & $\begin{array}{l}1.0 \\
0\end{array}$ & 5.48 & $\begin{array}{l}10 . \\
43\end{array}$ & 1090 & $\begin{array}{l}14 . \\
3\end{array}$ & $\begin{array}{l}4582 \\
4\end{array}$ & - & - & - & - & $\begin{array}{l}11.8 \\
57\end{array}$ \\
\hline 42 & 29.40 & 12.40 & 500 & $\mathrm{KH}$ & 6.26 & $\begin{array}{l}0.5 \\
1\end{array}$ & $\begin{array}{l}60.0 \\
5\end{array}$ & $\begin{array}{l}0.1 \\
31\end{array}$ & $\begin{array}{l}35.1 \\
4\end{array}$ & $\begin{array}{l}38 . \\
89\end{array}$ & $\begin{array}{l}4265 \\
0\end{array}$ & - & - & - & - & - & - & $\begin{array}{l}39.5 \\
8\end{array}$ \\
\hline 43 & 29.40 & 12.40 & 500 & $\mathrm{H}$ & $\begin{array}{l}120 . \\
4\end{array}$ & $\begin{array}{l}0.8 \\
1\end{array}$ & $\begin{array}{l}26.6 \\
7\end{array}$ & $\begin{array}{l}32 . \\
56\end{array}$ & 4000 & - & - & - & - & - & - & - & - & $\begin{array}{l}33.3 \\
66\end{array}$ \\
\hline 44 & 29.40 & 12.40 & 500 & $\mathrm{AA}$ & $\begin{array}{l}11.6 \\
1\end{array}$ & $\begin{array}{l}1.2 \\
0\end{array}$ & $\begin{array}{l}19.0 \\
0\end{array}$ & $\begin{array}{l}17 . \\
75\end{array}$ & $\begin{array}{l}116 . \\
9\end{array}$ & $\begin{array}{l}16 . \\
03\end{array}$ & $\begin{array}{l}4549 \\
4\end{array}$ & - & - & - & - & - & - & 19 \\
\hline 45 & 29.40 & 12.40 & 525 & AA & 1.83 & $\begin{array}{l}0.1 \\
3\end{array}$ & $\begin{array}{l}34.3 \\
6\end{array}$ & $\begin{array}{l}12 . \\
29\end{array}$ & $\begin{array}{l}37.9 \\
0\end{array}$ & $\begin{array}{l}28 . \\
71\end{array}$ & 9728 & - & - & - & - & - & - & $\begin{array}{l}41.1 \\
31\end{array}$ \\
\hline 46 & 29.40 & 12.40 & 525 & $\mathrm{QH}$ & 549 & $\begin{array}{l}0.3 \\
8\end{array}$ & $\begin{array}{l}44.2 \\
2\end{array}$ & $\begin{array}{l}1.5 \\
2\end{array}$ & $\begin{array}{l}25.5 \\
2\end{array}$ & $\begin{array}{l}29 . \\
69\end{array}$ & 2185 & - & - & - & - & - & - & $\begin{array}{l}31.5 \\
86\end{array}$ \\
\hline 47 & 29.40 & 12.40 & 525 & HA & $\begin{array}{l}43.8 \\
1\end{array}$ & $\begin{array}{l}0.3 \\
9\end{array}$ & 4.10 & $\begin{array}{l}0.3 \\
25\end{array}$ & $\begin{array}{l}19.2 \\
9\end{array}$ & $\begin{array}{l}20 . \\
86\end{array}$ & $\begin{array}{l}1575 \\
8\end{array}$ & - & - & - & - & - & - & $\begin{array}{l}21.5 \\
77\end{array}$ \\
\hline 48 & 29.70 & 12.60 & 534 & QHA & 1312 & $\begin{array}{l}1.6 \\
7\end{array}$ & $\begin{array}{l}239 . \\
4\end{array}$ & $\begin{array}{l}44 . \\
69\end{array}$ & 4.11 & $\begin{array}{l}67 . \\
62\end{array}$ & $\begin{array}{l}69.8 \\
2\end{array}$ & $\begin{array}{l}33 . \\
0\end{array}$ & 1000 & - & - & - & - & $\begin{array}{l}146 . \\
98\end{array}$ \\
\hline 49 & 29.70 & 12.60 & 534 & $\begin{array}{l}\text { HKQ } \\
\mathrm{H}\end{array}$ & $\begin{array}{l}281 . \\
6\end{array}$ & $\begin{array}{l}9.7 \\
7\end{array}$ & $\begin{array}{l}20.2 \\
3\end{array}$ & $\begin{array}{l}0.9 \\
75\end{array}$ & 6943 & $\begin{array}{l}1.6 \\
5\end{array}$ & $\begin{array}{l}58.3 \\
9\end{array}$ & $\begin{array}{l}15 . \\
6\end{array}$ & $\begin{array}{l}10.0 \\
9\end{array}$ & $\begin{array}{l}12 \\
0\end{array}$ & 1000 & - & - & $\begin{array}{l}147 . \\
92\end{array}$ \\
\hline 51 & 29.70 & 12.60 & 539 & QQH & 1242 & $\begin{array}{l}2.9 \\
7\end{array}$ & $\begin{array}{l}413 . \\
9\end{array}$ & $\begin{array}{l}8.1 \\
4\end{array}$ & $\begin{array}{l}209 . \\
6\end{array}$ & $\begin{array}{l}36 . \\
65\end{array}$ & 4.56 & $\begin{array}{l}57 . \\
2\end{array}$ & $\begin{array}{l}44.4 \\
7\end{array}$ & - & - & - & - & $\begin{array}{l}>10 \\
5\end{array}$ \\
\hline 52 & 29.40 & 12.50 & 597 & QHA & $\begin{array}{l}223 . \\
6\end{array}$ & $\begin{array}{l}0.8 \\
7\end{array}$ & $\begin{array}{l}21.8 \\
1\end{array}$ & $\begin{array}{l}8.3 \\
5\end{array}$ & 2.76 & $\begin{array}{l}5.8 \\
5\end{array}$ & $\begin{array}{l}58.6 \\
9\end{array}$ & $\begin{array}{l}10 \\
4\end{array}$ & $\begin{array}{l}6934 \\
.5\end{array}$ & - & - & - & - & $\begin{array}{l}15.6 \\
6\end{array}$ \\
\hline 53 & 29.40 & 12.50 & 606 & QHK & 1457 & $\begin{array}{l}1.0 \\
9\end{array}$ & $\begin{array}{l}183 . \\
1\end{array}$ & $\begin{array}{l}2.6 \\
2\end{array}$ & $\begin{array}{l}26.3 \\
8\end{array}$ & $\begin{array}{l}5.8 \\
6\end{array}$ & $\begin{array}{l}448 . \\
6\end{array}$ & $\begin{array}{l}63 . \\
7\end{array}$ & $\begin{array}{l}35.9 \\
9\end{array}$ & - & - & - & - & $\begin{array}{l}73.3 \\
3\end{array}$ \\
\hline 54 & 29.40 & 12.50 & 606 & $\mathrm{QH}$ & $\begin{array}{l}492 . \\
8\end{array}$ & $\begin{array}{l}1.5 \\
3\end{array}$ & $\begin{array}{l}20.3 \\
3\end{array}$ & $\begin{array}{l}40 . \\
20\end{array}$ & 6.44 & $\begin{array}{l}69 . \\
01\end{array}$ & 4000 & - & - & - & - & - & - & $\begin{array}{l}110 . \\
74\end{array}$ \\
\hline 55 & 29.00 & 12.90 & 530 & HK & 2080 & $\begin{array}{l}3.7 \\
4\end{array}$ & $\begin{array}{l}101 . \\
5\end{array}$ & $\begin{array}{l}70 . \\
01\end{array}$ & $\begin{array}{l}437 . \\
3\end{array}$ & $\begin{array}{l}77 . \\
93\end{array}$ & $\begin{array}{l}27.0 \\
2\end{array}$ & - & - & - & - & - & - & $\begin{array}{l}>20 \\
0\end{array}$ \\
\hline 56 & 28.70 & 12.90 & 610 & $\begin{array}{l}\text { QHA } \\
\mathrm{K}\end{array}$ & $\begin{array}{l}219 . \\
5\end{array}$ & $\begin{array}{l}1.0 \\
9\end{array}$ & 75.5 & $\begin{array}{l}4.4 \\
4\end{array}$ & 7.39 & $\begin{array}{l}2.3 \\
0\end{array}$ & $\begin{array}{l}97.3 \\
5\end{array}$ & $\begin{array}{l}10 \\
2\end{array}$ & $\begin{array}{l}784 . \\
2\end{array}$ & $\begin{array}{l}65 . \\
9\end{array}$ & $\begin{array}{l}76.0 \\
0\end{array}$ & - & - & $\begin{array}{l}>20 \\
0\end{array}$ \\
\hline 57 & 28.70 & 12.90 & 610 & $\begin{array}{l}\text { QHA } \\
\mathrm{K}\end{array}$ & $\begin{array}{l}257 . \\
7\end{array}$ & $\begin{array}{l}0.9 \\
8\end{array}$ & 104 & $\begin{array}{l}3.8 \\
5\end{array}$ & $\begin{array}{l}13.9 \\
5\end{array}$ & $\begin{array}{l}2.1 \\
4\end{array}$ & $\begin{array}{l}71.2 \\
5\end{array}$ & $\begin{array}{l}34 . \\
4\end{array}$ & $\begin{array}{l}324 . \\
7\end{array}$ & $\begin{array}{l}93 . \\
0\end{array}$ & $\begin{array}{l}72.2 \\
3\end{array}$ & - & - & $\begin{array}{l}>20 \\
0\end{array}$ \\
\hline 58 & 28.60 & 12.90 & 613 & $\begin{array}{l}\text { QQQ } \\
\mathrm{H}\end{array}$ & 4566 & $\begin{array}{l}0.8 \\
1\end{array}$ & 1353 & $\begin{array}{l}3.7 \\
1\end{array}$ & $\begin{array}{l}175 . \\
5\end{array}$ & $\begin{array}{l}18 . \\
70\end{array}$ & $\begin{array}{l}34.3 \\
1 \\
\end{array}$ & $\begin{array}{l}81 . \\
9\end{array}$ & 2.69 & $\begin{array}{l}28 . \\
9\end{array}$ & 6235 & - & - & $\begin{array}{l}133 . \\
99\end{array}$ \\
\hline
\end{tabular}

Table 5: Calculated Values of " $R_{\mathrm{f}}$ ", " $\mathrm{R}_{\mathrm{w}}$ ", "F" and "$\Phi$ " for Some Locations in the Study Area

\begin{tabular}{|c|c|c|c|c|c|c|c|}
\hline No. & Location & $\begin{array}{l}\text { COORDINATES } \\
\text { Easting }\end{array}$ & Northing & $\mathrm{R}_{\mathrm{f}} \mathrm{Ohm} \cdot \mathrm{m}$ & $\mathrm{R}_{\mathrm{w}} \mathrm{Ohm} \cdot \mathrm{m}$ & $\mathrm{F}$ & $\Phi$ \\
\hline 1 & El Khuwei & 29.20 & 13.10 & 131.0 & 26.39 & 4.96 & 33.50 \\
\hline 2 & Ankosh & 29.10 & 13.00 & 97.03 & 30.30 & 3.20 & 25.94 \\
\hline 4 & El Howag & 28.80 & 12. 60 & 442.2 & 64.90 & 6.80 & 40.24 \\
\hline 5 & Kamas Hallab & 28.90 & 12.70 & 164.0 & 65.36 & 3.51 & 27.4 \\
\hline 6 & Khamas El Donkey & 29.91 & 12.75 & 62.48 & 33.70 & 1.85 & 18.88 \\
\hline 7 & Gar Um Bilbil & 29.20 & 12.50 & 52.00 & 30.13 & 1.73 & 18.16 \\
\hline 8 & El Doodiya & 29.36 & 13.03 & 232.0 & 30.33 & 7.65 & 43.10 \\
\hline
\end{tabular}

The thickness of the superficial also varies between apportion of a meter to about 47 meters.

The second unit, the Nubian Sandstone Formation which is relatively large in thickness and ranges from few meters at the extremity of the basin to more than 300 meters within the basin, and having resistivity ranges from few to thousands ohm.m. The higher values usually correspond to silicified sandstones and conglomerates. The water saturated zones in the Nubian show resistivity range as 18- 479 ohm.m and thickness range from 12- $140 \mathrm{~m}$. Porosity calculated for these zones have values range from 1.88-
50.9\% (table 4). The Nubian Sandstone Formation is absent in some locations, this is at the periphery of the basin, where the Superficial Deposits rests directly on the Basement Complex. In these cases the weathered Basement Complex is relatively shallow and at times represents the aguiferous zone (Fig. 8). 


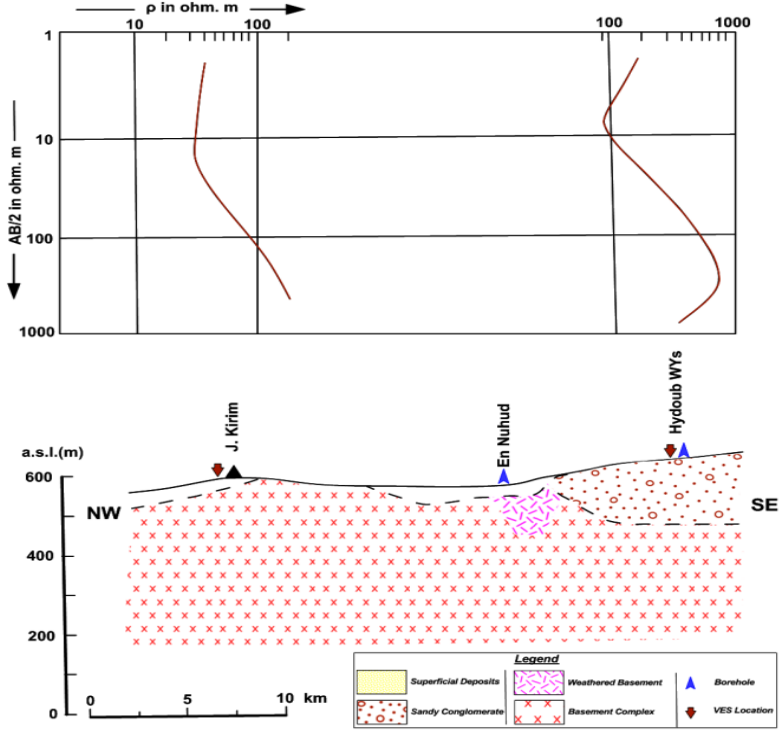

Fig. 8: Basement Complex as Aquiferous Zone.

The Basement complex is not usually showed in VES measurements due to the thick sedimentary sequence and limitation of the resistivity method. The Basement Complex shows resistivity range as $36-\infty$ ohm.m, according to their mineralogy and the degree of weathering. The thickness and degree of weathering are

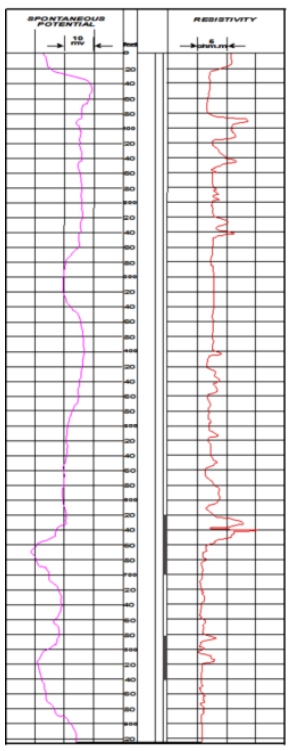

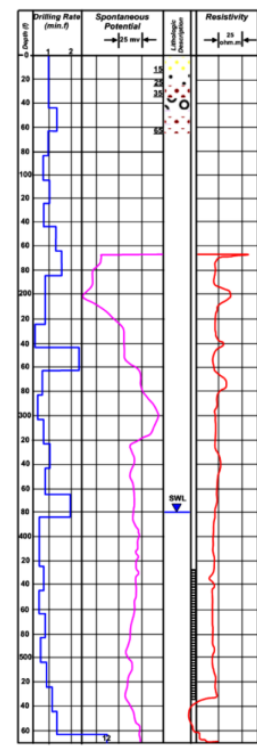

(b) highly variable and most probably shares the groundwater storage with the upper sediments.

\subsection{Geophysical well logs}

The depth of the logged boreholes ranges from 52 to $280 \mathrm{~m}$. All these boreholes were partially penetrated by geophysical logging except the Well log at El Mayaa and the drilling time log at Kul Yusuf. Formation resistivity for the aquiferous zones from the logs (Fig. 9) ranges from 5 to $48 \mathrm{ohm} . \mathrm{m}$, and from 5 to 189 for SP (table 6).

The values of resistivity and SP are not corrected for the influence of the drilling fluids, where there is no data about the characteristics of these fluids; hence these values are used just as representatives for the lithological characteristics. The range of these values indicate that some parts of the aquiferous zones are intercalated with fine materials.

Two drilling time logs were measured in hasabenabi in the western part of the basin and Kul Yusuf at the north eastern corner (Fig. 10). In hasabenabi (T.D.: $162 \mathrm{~m}$ ) the drilling time rate is measured from the half depth to the bottom (loss of circulation: 18 $\mathrm{m}$ to bottom). The drilling time rate is clearly slow and highly variable at the non-saturated zone, and relatively fast at the saturated zone. At Kul Yusuf borehole (T.D.: 90m), three distinct zones are distinguished; the first zone with relatively fast drilling rate, it is normally the

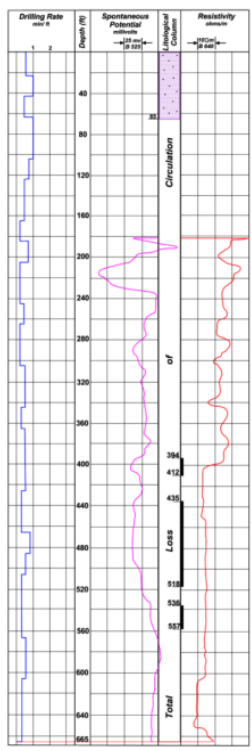

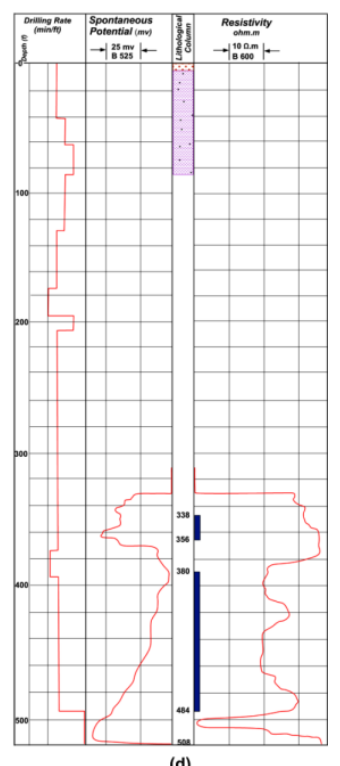

Fig. 9: Geophysical Well Logs: (A) El Mayaa, (B) Munem, (C) El Tugur, (D) El Khuwei.

Table 6: Summary of the Geophysical Well Logs

\begin{tabular}{llllllll}
\hline$\#$ & Location & T. Depth $(\mathrm{m})$ & $\begin{array}{l}\text { Logging } \\
\text { Depth }(\mathrm{m})\end{array}$ & $\begin{array}{l}\text { Saturated } \\
\text { Thick. }(\mathrm{m})\end{array}$ & F. Res. $(\mathrm{m})$ & $\begin{array}{l}\text { SP }(\mathrm{mv}) \\
\text { quality }\end{array}$ & Remarks \\
\hline 1 & El Khuwei & 155 & $100-155$ & 52 & $30-48$ & $5-63$ & Fresh \\
2 & Et Tugur & 203 & $54-203$ & 125 & $15-35$ & Loss of circulation \\
3 & Munim & 174 & $51-174$ & 112 & $17-38$ & $68-189$ & Fresh \\
4 & El Mayaa & 280 & $0-280$ & 109 & $5-15$ & Foss of circulation \\
Loss of circulation & Fresh & - \\
\hline
\end{tabular}




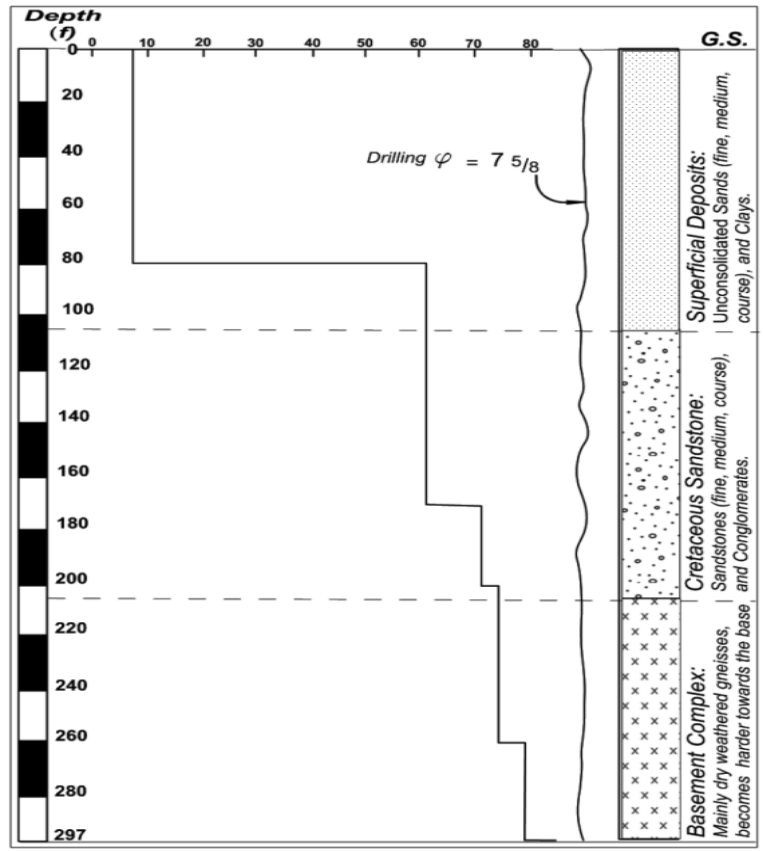

(a)

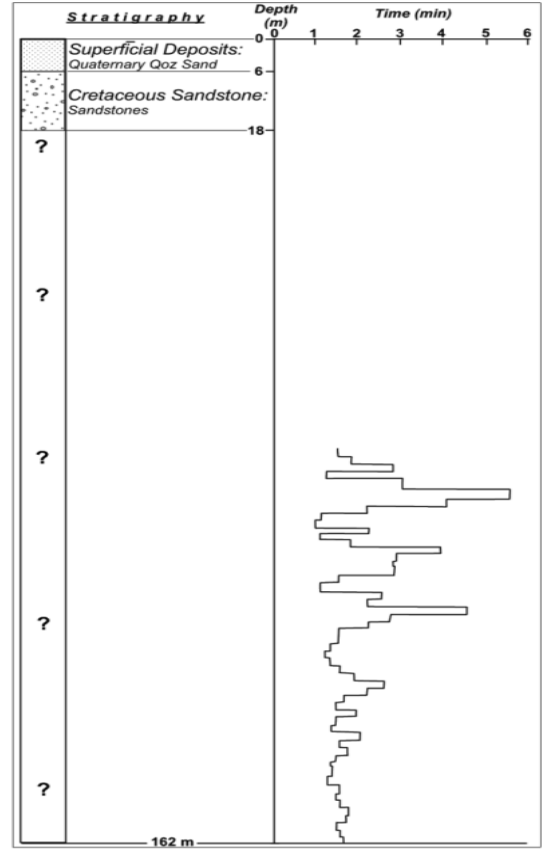

(b)

Superficial Deposits, the second with relatively medium rate, corresponds to Nubian Sandstone Formation, and the third with high and variable rates is compared with the Basement Complex of variable weathering degree, and part of the Nubian Sandstone which may be of silicified cement.

\section{Integrated interpretation of geophysical da- ta}

Combination of gravity, magnetic and geoelectric measurements give good results, where the gravity and magnetic data determine the depth to the Basement and geoelectric data clearly resolve the layering and lithology variations and serve on determining the weathering zones in the Basement rocks (Fig. 11).

According to quantitative interpretation of gravity integrated with geomagnetic and borehole data, it is revealed that the thickness of sediments is varied and reaches about 1000 meters at Gar Um Bilbil depression (Fig. 4).

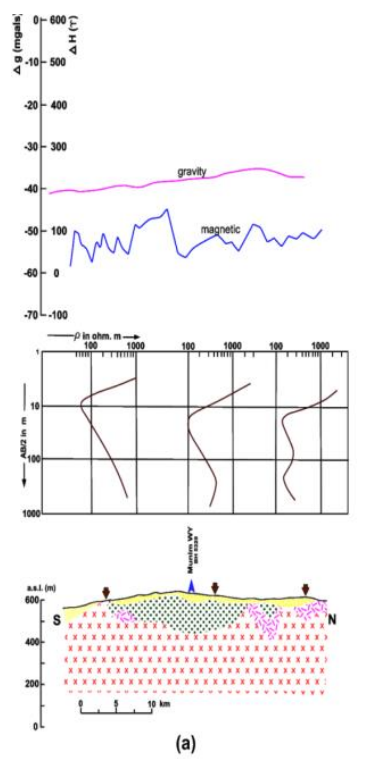

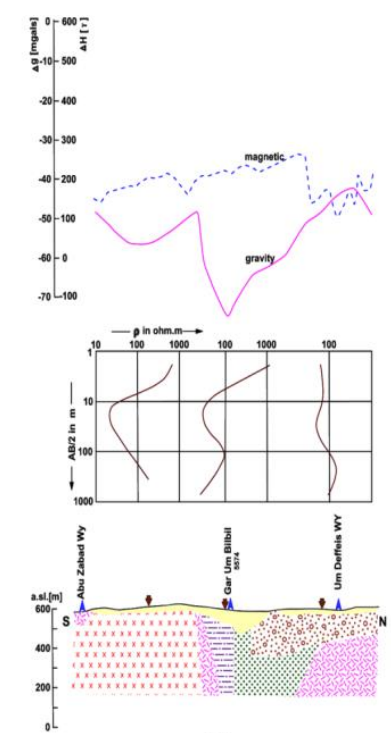

(b)

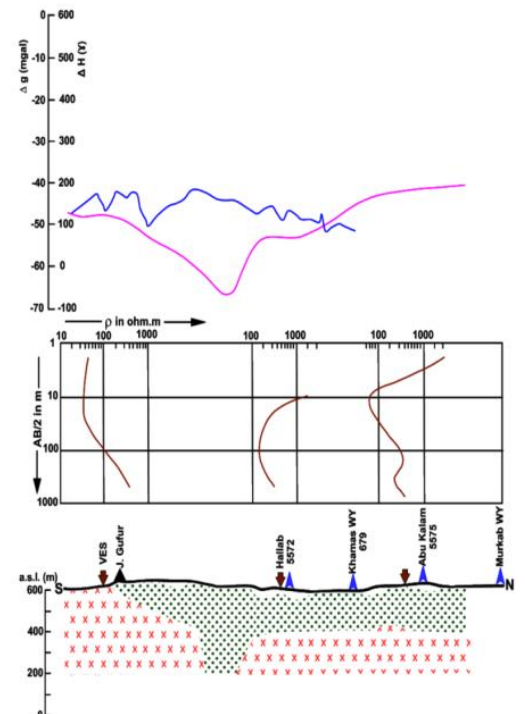

(c)

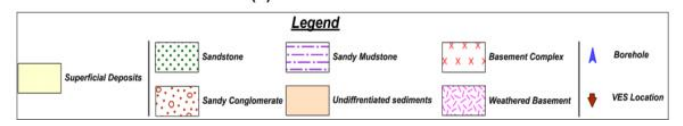

Fig. 11: Combination of Geophysical Data Gravity, Magnetic and Rasistivity. 


\section{Conclusion}

Interpretation of geophysical data indicates that En Nuhud Basin is structural depression as a half-graben produced by displacements along a system of faults. three stratigraphic units occupy the depression; the Superficial Deposits, Nubian Sandstone Formation, and the Basement Complex. The Superficial Deposits at the surface of the sequence which is mostly of sandy materials have a thickness varies between apportion of a meter to about 47 meters. The second unit is the Nubian Sandstone Formation which is relatively large in thickness and ranges from few meters at the extremity of the basin to more than 300 meters within the basin. The water saturated zones in the Nubian show good characteristics and a thickness range from 12- $140 \mathrm{~m}$. The Nubian Sandstone Formation is absent in some locations, this is at the periphery of the basin, where the Superficial Deposits rests directly on the shallow Basement Complex. The weathered Basement Complex is sometimes represents the aguiferous zone.

\section{Acknowledgement}

Acknowledgement to the colleagues and practitioners in the relative institutes and agencies for allowing their own archive data and for some technical support in processing and analyzing the data of this study.

\section{References}

[1] Dobrin, M.B., (1988). Introduction to Geophysical Prospecting Fourth Edition, McGRaw-Hill Book Company, Singapore for manufacture and export.

[2] Geological Map of Sudan (1981). Prepared by G.M.R.D. Khartoum- Sudan, the Assistance of R.S.C. Geddah- Kingdom of Saudi Arabia and Collaboration of the B.R.G.M. Orleans France.

[3] Ginaya, M. A., (2001). Hydrogeological Investigation of En Nuhud Basin, West Kordofan State, Sudan. MSc Thesis, Department of Geology and Mining, College of Natural Resources and Environmental Studies, Juba University, Sudan.

[4] Ginaya, M. A., (2011). Updating of Groundwater Conditions of Ennahud Basin, North Kordofan, Sudan. PhD Thesis, Institute for Energy \& Earth Sciences, Ministry of Sciences \& Technology, Sudan.

[5] Harms, U., Schandelmeier, H. and Darbyshire, D. P (1990). PanAfrican Reworked Early/Middle Proterozoic Crust in NE Africa West of the Nile: Sr and Nd Isotope Evidance. Journal of the Geological Society, London, Britain, Vol. 147, PP 859-872. https://doi.org/10.1144/gsigs.147.5.0859.

[6] Karkanis, B.G. (1966). Hydrochemical Facies of Grounwater in the Western Provinces of Sudan. MSc. Thesis, Graduate College, University of Arisona.

[7] Kearey, P., Brooks M. and Hill, I. (2002). An Introduction to Geophysical Exploration, third edition. Blackwell Science Ltd, London, Britain.

[8] Keller, G. V., and Frischknecht, F. C., 1966, Electrical methods in geophysical prospecting, v. 10 of International Series of Monographs in Electromagnetic Waves: New York, Pergamon Press.

[9] Kirsch, R., (2006). Groundwater Geophysics, a Tool for Hydrogeology. Springer-Verlag Berlin Heidelberg 2006 Printed in Germany.

[10] Keys, W.S. \& MacCarry, L.M., (1981). Application of Borehole Geohysics to Water Resources Investigations, Book 2, Colliction of Environmentl Data, Chapter E1. Third Printing. Techniques of Water Resources Investigation of United States Geological Survey.

[11] Rodis, H.G., Hassan, A. \& Wahadan, L., (1964). Grounwater Geology of Kordofan Province, Pulletin No. 14. Minstry of Mineral Resources, Geologica Survey Department, Khartoum, Sudan.

[12] Strojexport (1971-1972). Parametrical Measurements at Boreholes, Appendix 1.

[13] Strojexport (1971-1972). Log description with Geophysical Parameters, Appendix 4 .

[14] Strojexport (1971-1972). Catalog of Gravity Values, Appendix 6.

[15] Strojexport (1972). Groundwater Research, SW part of Kordofan, Second Stage, Final Report, Praque.
[16] Strojexport (1973). Preliminary Interpretation of the first part of the Third Stage Geophysical Investigation in the Western and Central part of Kordofan Province in the Sudan. The Foreign Trade Corporation Strojexport, PRAHA 1, Czechslovakia.

[17] Strojexport (1975). Geophysical Investigation of Hydrogeological Structures, Western Part of Kordofan Province.

[18] Strojexport (1975). Parametrical Measurements at Boreholes, Appendix 1.

[19] Strojexport (1975). Geoelectric Resistivity Sections with Gravity and Magnetic Curves, Appendix 2.

[20] Strojexport (1975). Log Description of Boreholes, Appendix 5.

[21] Strojexport (1975). Parametrical Analysis, Appendix 7.

[22] Strojexport (1976). Interpretative Gravity Profiles and Geoelectric Resistivity Sections Including Gravity and Magnetic Graphs, Appendix 4.

[23] Strojexport (1976). Geophysical Investigations of Groundwater Structures, Western Part of Kordofan Province an Eastern Part of Darfur Province, Fourth Stage. Strojexport Foreign Trade Corporation for Export and Import, Praque, Czechoslovakia.

[24] Vail, J.R., (1978). Outline of the geology and Mineral Resources of the Democratic Republic of the Sudan and Adjacent Areas. Overseas Geology \& Minerel Resources No. 49.

[25] Whiteman, A.G., (1971). The Geology of the Sudan Rpublic. Clarendon Press, Oxford, England. 